\title{
High-Flow Nasal Cannula: Clinically Speaking, Is There Really a Difference?
}

Historically, oxygen nasal cannulae are used as a therapy to increase arterial oxygenation in patients who are hypoxemic. In the neonatal practice, oxygen flows of $>2 \mathrm{~L} / \mathrm{min}$ and of $>4 \mathrm{~L} / \mathrm{min}$ in the pediatric patient have seldom been used due to the potential for excessive drying of the nasal mucosae and risk for developing hypothermia. It has been speculated that larger pediatric patients and adult patients are able to better tolerate flows in excess of $4 \mathrm{~L} / \mathrm{min}$ because the large nasal mucosal surface area can support hydration of dry medical grade gases. High-flow nasal cannula (HFNC) therapy is a form of respiratory support that provides flows believed to be in excess of a patient's spontaneous inspiratory and expiratory flows. In the past decade, a proliferation of heated, humidified HFNC devices have been introduced into the clinical setting. These devices are intended to provide optimal heating and humidification of medical gases regardless of the flow setting. In the case of neonates, which have been the predominant pediatric population that receives HFNC, there is no clear consensus on which flows constitute "high" in this population. If "high" is intended to mean a flow that exceeds spontaneous inspiratory flow, then there is no reasonable clinical measurement to ascertain this relationship. Thus, the true definition of HFNC remains elusive.

One neonatal source defines HFNC as flows $>1 \mathrm{~L} / \mathrm{min}$ and another defines flows $>3 \mathrm{~L} / \mathrm{min}$ as HFNC. ${ }^{1}$ Classification is further complicated in larger pediatric patients and in adult patients when flows during HFNC have been reported to be in excess of those traditionally used with a standard nasal cannula $(\sim 6 \mathrm{~L} / \mathrm{min})$ and as high as $30-$ $40 \mathrm{~L} / \mathrm{min}^{2}{ }^{2}$ With the technological ability to provide better heat and humidity, clinicians have found that HFNC may be able to support a larger fraction of patients who would otherwise require CPAP, noninvasive ventilation, or invasive mechanical ventilation. There are several proposed mechanisms by which HFNC may provide greater respiratory assistance than standard oxygen delivery devices.

Infants have a significant amount of upper airway resistance (particularly at the nasal level), designed to help maintain open airways and, therefore, functional residual capacity during exhalation. ${ }^{3}$ Flows provided by HFNC in

The author has disclosed no conflicts of interest.

DOI: $10.4187 /$ respcare.06794 excess of the inspiratory flows may offer inspiratory assistance by overcoming resistance of the upper airway. Flows in excess of the expiratory flow may provide "back pressure" at the nasal airway opening during exhalation,

See the Original Study on Page 10

similar to grunting or positive expiratory pressure therapy. This may assist with maintaining or increasing functional residual capacity. In addition, gases provided at body temperature and pressure saturated that exceed expiratory flows may provide a physiologic purging of carbon dioxide from the anatomic dead space via anatomic leak (nasal-oral airway) while maintaining homeostasis of the upper airway and elevating the faction of inspired oxygen. These effects are likely to vary based on the flow, minute ventilation, patient size (extrathoracic dead space), leak, and nasal airway opening-prong size relationship. Clinical acceptance is related to the fact that HFNC is less expensive, simpler to operate, and requires a less-complicated airway interface than a standard CPAP or noninvasive ventilation device. Another proposed benefit is that HFNC prongs are generally less occlusive and may cause less skin or nasal injury than prongs or a mask. The widespread acceptance and use of this approach have been implemented with very little experimental data to support HFNC flow settings as a safe and effective option in all patients with respiratory insufficiency.

In this issue of Respiratory CARE, Itagaki et al ${ }^{4}$ described the effects of oronasal mask oxygen therapy when compared with 1 and $2 \mathrm{~L} / \mathrm{kg} / \mathrm{min}$ of HFNC on thoraco-abdominal synchrony in 10 subjects with mild-to-moderate respiratory failure after cardiac surgery. Their goal was to determine optimal flows of HFNC within a pediatric cohort of subjects who weighed $2-10 \mathrm{~kg}$. This study built on their previous reports on the effects of HFNC on thoraco-abdominal synchrony. ${ }^{5}$ The investigators concluded that HFNC at $2 \mathrm{~L} / \mathrm{kg} / \mathrm{min}$ improved thoraco-abdominal synchrony and reduced breathing frequency. ${ }^{4}$ However, it remains unclear as to whether $2 \mathrm{~L} / \mathrm{kg} / \mathrm{min}$ is clinically superior.

The definitions of respiratory failure are unusual. General definitions of respiratory failure are often broken down into 2 threshold types. After those thresholds are exceeded, then mild, moderate, and severe can be classified. Type I or hypoxemic respiratory failure is often defined as $\mathrm{P}_{\mathrm{aO}_{2}}$ 


\section{EDITORIALS}

$<60 \mathrm{~mm} \mathrm{Hg}\left(\mathrm{S}_{\mathrm{pO}_{2}}<90 \%\right)$. In this study, it was defined as $\mathrm{S}_{\mathrm{pO}_{2}}<95 \%$. Type II or hypercapnic respiratory failure is often defined by a $\mathrm{PaCO}_{2}$ of $>45 \mathrm{~mm} \mathrm{Hg}$; yet, in this study, it was defined as a breathing frequency of $>50$ breaths $/ \mathrm{min}$ and/or the presence of asynchronous or a paradoxical breathing pattern. Normal frequencies for the subjects studied would be $30-60$ breaths/min and asynchronous or paradoxical breathing in this population is fairly common and very difficult to quantify by traditional physical assessment. When using the strict definition of respiratory failure, these subjects had respiratory distress or insufficiency, but not failure. Subjects who had true respiratory failure $\left(\mathrm{P}_{\mathrm{aCO}_{2}}>60 \mathrm{~mm} \mathrm{Hg}\right.$; $\mathrm{P}_{\mathrm{aO}_{2}} / \mathrm{Fi}_{\mathrm{O}_{2}}<150$ and $\mathrm{pH}<7.20$ ) were excluded. I highlight this because definitions are important and ensure proper interpretations of results.

One subject was enrolled for hypoxemic respiratory failure, and 2 subjects were enrolled for tachypnea. None of the subjects had $\mathrm{P}_{\text {aCO }}>40 \mathrm{~mm} \mathrm{Hg}$ or a frequency $>45$ breaths/ min. The majority of infants included (70\%) were found to have asynchronous or paradoxical breathing. This is important to note because the primary outcome measure was thoraco-abdominal synchrony by Respiratory Inductance plethysmography and may demonstrate a selection bias.

From a clinical perspective, a reduction in frequency of 3-4 breaths/min when study subjects are already breathing on the lower threshold of normal would be seen as insignificant. There was only a decrease by 2 breaths/min from HFNC set at 1 to $2 \mathrm{~L} / \mathrm{kg} / \mathrm{min}$. The reduction in phase angle (surrogate of synchrony) looks impressive and certainly would contribute to our understanding of HFNC, yet, if you were to compare it with control 2, then all effect is lost. In fact, if the breathing frequency were compared with control 2, I am not sure that it would be statically significant. If there was a positive pressure effect created by the HFNC intervention that increased functional residual capacity or the efficiency of the respiratory muscles, then the tidal volume would have increased, therefore, causing the patient to lower his or her breathing frequency to maintain alveolar ventilation:

Alveolar ventilation $=($ tidal volume - dead space $)$

$$
\times \text { breathing frequency }
$$

If $\mathrm{P}_{\mathrm{aCO}}$ remained the same, then one could assume that alveolar ventilation remained constant given the same metabolic rate. If alveolar ventilation is constant and the investigators reported a minute ventilation decrease after the application of HFNC, then one can assume that dead space was the major contributing factor. When exploring a little deeper, we read that the decrease in breathing frequency was not solely responsible for the decrease in minute ventilation. Tidal volume decreased, although not significantly. The reduction of dead space improved respiratory efficiency and led to a natural response and reduction in respiratory drive.
The choice to use $2 \mathrm{~L} / \mathrm{kg} / \mathrm{min}$ based on current practice and previous studies was reasonable but should also be based on estimated flows and breathing patterns within the study subjects. When speaking physiologically, $2 \mathrm{~L} / \mathrm{kg} / \mathrm{min}$ far exceeds inspiratory and certainly expiratory flows almost by two. If you take a tidal volume of $8 \mathrm{~mL} / \mathrm{kg}$ (the range in this study was $7-8.2 \mathrm{~mL} / \mathrm{kg}$ ) with a $33 \%$ inspiratory time and the highest normal breathing frequency, $1-1.2 \mathrm{~L} / \mathrm{kg} / \mathrm{min}$ should be enough to overcome inspiratory flows. Yet, this cannot be linearly applied because as patients age, their breathing patterns and flows change disproportionally to weight. The lack of substantial improvement from 1 to $2 \mathrm{~L} / \mathrm{kg} / \mathrm{min}$ led me to question whether $2 \mathrm{~L} / \mathrm{kg} / \mathrm{min}$ is superior to $1 \mathrm{~L} / \mathrm{kg} / \mathrm{min}$. I certainly would not recommend starting at $2 \mathrm{~L} / \mathrm{kg} / \mathrm{min}$ and titrating up if distress did not subside because you run the risk of not escalating to invasive mechanical ventilation appropriately.

This study leads us to extrapolate a few additional relationships. The lowering of the frequency and improvement in the phase angle were likely the effect of a lower work of breathing from the efficiency gained in respiration by using HFNC. These effects are seen in patients with respiratory insufficiency or distress. The work by Itagaki et $\mathrm{al}^{4}$ has given us yet another piece of the puzzle to consider. However, due to marginal improvements and the low number of subjects enrolled, I remain skeptical that HFNC truly improves thoraco-abdominal synchrony. I remain convinced that HFNC provides support through improvement in respiratory efficiency through a reduction in dead space, not just because of this study, but because it is consistent with other higher-level evidence. I hope the investigators and readers will take these methods and apply them to a larger population of patients with mild or moderate respiratory failure. Only then will we be able to truly know the effects of HFNC therapy on thoraco-abdominal synchrony in pediatric patients.

Brian K Walsh

Respiratory Therapy

Liberty University

Lynchburg, Virginia

\section{REFERENCES}

1. Wilkinson D, Andersen C, O'Donnell CP, De Paoli AG. High flow nasal cannula for respiratory support in preterm infants. Cochrane Database Syst Rev 2011(5):CD006405.

2. Urbano J, del Castillo J, López-Herce J, Gallardo JA, Solana MJ, Carrillo Á. High-flow oxygen therapy: pressure analysis in a pediatric airway model. Respir Care 2012;57(5):721-726.

3. Doershuk GF, Matthews LW. Airway resistance and lung volume in the newborn infant. Pediatr Res 1969;3(2):128-134.

4. Itagaki T, Nakanishi N, Okuda N, Nakataki E, Onodera M, Oto J, et al. Effect of high-flow nasal cannula on thoraco-abdominal synchrony in pediatric subjects after cardiac surgery. Respir Care 2018.

5. Itagaki T, Okuda N, Tsunano Y, Kohata H, Nakataki E, Onodera M, et al. Effect of high-flow nasal cannula on thoraco-abdominal synchrony in adult critically ill patients. Respir Care 2014;59(1):70-74. 